\title{
TECHNIQUES FOR THE STUDY OF ANT LARVAE
}

\author{
By George C. Wheeler and Jeanette Wheeler \\ University of North Dakota, Grand Forks
}

Our studies on ant larvae have been published in 30 separate articles scattered in eight periodicals, appearing over a period of 33 years. Several entomologists have expressed regret that the studies have not been combined into a monograph. We regret this too, but no foundation has been willing to publish so long a treatise with so many illustrations and without economic or popular appeal, and no journal has been willing to accept it on the installment plan. The exigency of finance, then, has forced us to publish the body of the "monograph" piecemeal. This present article can be regarded as a part of the introduction. We are prompted to publish it at this time by requests for information concerning our techniques.

\section{Living Material}

When living larvae are available, we observe and record as much as possible of their external anatomy. If the larvae are active the changeability of form is noted.

\section{Preservation}

Any preservative may be used for ant larvae if only the external anatomy is to be studied. We prefer $85 \%$ ethyl alcohol. No special preparation is necessary; the larvae are simply dropped into the preservative. If the alcohol is too dilute or if the container has been overcrowded internal structures may be inadequately preserved, but the integument will still be usable for external anatomy. If large larvae are jostled the hairs may get broken.

Naturally if one plans to investigate also internal anatomy or histology, appropriate killing and fixing reagents are necessary.

If we have only one larva (or very few) of a species we make notes and drawings before subjecting it to any additional techniques. This is simply good insurance.

\section{Relaxation}

Even dried larvae may be used for the study of external anatomy. Either of two methods of relaxing is recommended: 
I. Two days in a $0.5 \%$ solution of trisodium phosphate. Transfer to $85 \%$ alcohol.

2. Two days (or less) in the following - 95\% ethyl alcohol 280 $\mathrm{ml}$; distilled water $230 \mathrm{ml}$; benzol $35 \mathrm{ml}$; ethyl acetate $95 \mathrm{ml}$.

Dried larvae are handled with extreme care, since hairs are more likely to get broken in this condition.

\section{Handling}

The most convenient receptacle for the treatment of larvae is a staining dish (= embryological cup) 4I X 4I X I $8 \mathrm{~mm}$ in outside dimensions. Minute specimens, however, are best treated in culture slides (= hanging-drop slides). Small specimens are transferred with a pipette, larger specimens between the points of forceps (but without compression). Or, if one prefers, the larvae may be left in the same dish or culture slide; the old reagent is drawn off with a pipette and then replaced with the next reagent.

\section{Cleaning}

The best cleaning reagent is potassium hydroxide solution ( IO gm $\mathrm{KOH}$ in $90 \mathrm{ml}$ water). While still in preservative the larvae are punctured with a dissecting needle or minute insect pin on the right side to permit the ready penetration of the cleaning solution. The number and locations of the punctures and the size of the needle depend upon the size of the larva. The specimen is left in the cleaning solution until all the internal tissues are dissolved and only a transparent exoskeleton remains.

If cleaning is not complete by the end of two days, the following procedure may prove effective: transfer to I \% hydrochloric acid and leave I 5 minutes; I 5 minutes in $95 \%$ alcohol; then leave in $\mathrm{KOH}$ until clean. Some larvae contain droplets of opaque substances which are insoluble in $\mathrm{KOH}$; these usually disappear later in alcohol or xylol.

\section{Staining}

The exoskeletons of ant larvae are stained in a very dilute solution of acid fuchsin. We use the following formula as a stock solution: acid fuchsin O.I gm, concentrated hydrochloric acid I ml, distilled water I liter; a few thymol crystals are added to prevent mold. The cleaned exoskeletons are washed in water for 15 minutes and transferred to $2 \mathrm{ml}$ of $1 \% \mathrm{HCL}$ in a staining dish; five drops of the stock solution of acid fuchsin are added. The integuments are left in the stain for 12 hours. We have experimented with stronger solutions of stain for shorter periods but have not been satisfied with the results. 
If overstained the integument can be completely decolorized by leaving it in a I \% solution of potassium hydroxide for several hours. It can then be washed and restained.

\section{Dehydration}

After staining, the exoskeletons are transferred to $95 \%$ alcohol (about $3 \mathrm{ml}$ ), in which they remain for about an hour. At the end of this period eight drops of carbolxylol (= 3 parts xylol and I part melted phenol crystals) are added every five or ten minutes until 48 drops have been added to the alcohol. If the carbolxylol is added too rapidly the exoskeletons may collapse and become distorted; they can be restored by transfer to $95 \%$ alcohol. If oil droplets separate from the alcohol, too much water has been carried over from the stain; the integuments are transferred to fresh $95 \%$ alcohol.

\section{Clearing}

From the mixture of carbolxylol and alcohol the integuments are transferred to pure carbolxylol and left for 15 to 60 minutes; then to xylol in a culture slide (or if large, in a staining dish). Add a drop of thin Canada balsam every I 5 minutes, allowing the xylol to evaporate meanwhile.

\section{Mounting}

When the balsam in the culture slide attains the same consistency as that which is being added, a large drop of the latter is placed on a slide and the exoskeleton is transferred to it. At this time medium to large specimens are surrounded - or at least flanked — by supports of some sort. We use fragments of broken slides which are of about the same thickness as the depth of the specimen. If it is obvious that the exoskeleton will not remain in the desired position, supports (such as a fine glass rod or fragments of cover slip) may be placed under the low part.

\section{Positioning}

With needles dipped in $x y l o l$ the integument is now arranged in the proper position for drawing (discussed below). Since the balsam must be soft at this stage, the specimen will rarely remain in precisely the desired position; hence the slide is allowed to rest flat in a covered container (e.g., a petri dish) for 24 hours.

If the specimen then needs rearranging, this is done under a stereomicroscope with needles dipped in xylol. If the balsam is too firm a drop of xylol is put on it and let stand a few minutes before using needles. If it is apparent that the specimen will not remain in the 
desired position, supports (fine glass rods or fragments of cover slip) may be inserted at this time. If supports were placed earlier, they may need readjustment. Shifting the retaining walls will sometimes correct the position of a specimen. After the rearrangement is completed more balsam is added if necessary to cover the specimen.

It is sometimes necessary to rearrange a specimen on half a dozen successive days. Once a specimen has remained in the desired position for 48 hours and is completely covered with balsam, the technique is considered finished.

If material is abundant we regularly make two kinds of mounts of each size and caste of each species: ( I ) an entire exoskeleton in side view; (2) detached head in full-face view, with the remainder of the exoskeleton ventral side up (or, if the head is applied to the ventral surface, the whole exoskeleton ventral side up). Other kinds of mounts are made whenever necessary.

For a side view an entire exoskeleton with the left side up and in strict profile, i.e., the middorsal line, the midventral line, the middle of the anus and the middle of the labium are all in the same plane. A good check is to be sure that the left spiracle of each somite is directly above the right spiracle of the same somite. It is conventional to show the entire larva viewed from the left side. Actually it doesn't matter which side is up. If for any reason (e.g., damaged left side) an integument is mounted with the right side up, it is a simple matter to reverse the original drawing.

The exoskeleton of the head is mounted in full-face (i.e., anterior) view. To accomplish this the uppermost line (imaginary) of the occipital border is set in the same plane as the lowermost part of the ventral surface of the labium.

When the head is applied to the ventral surface of the body (i.e., no neck), it is often possible to get it in the correct position by mounting an entire integument ventral side up. In most genera, however, it is necessary to cut off the head (either alone or with a portion of the prothorax) and mount it separately.

It is often necessary to prop up the ventral part of the head on fragments of cover slip, but only very large heads require retaining walls.

After the head is removed, the remainder of the integument is mounted with the ventral side up by following the procedure for the entire integument (see above). 


\section{Covering}

It is not, however, necessary to cover a mount if it is to be studied at low magnifications. In fact, covering is avoided whenever possible; if the balsam is soft (and it may take years for the interior of a thick mount to harden), the weight of the cover slip will set up currents which are likely to move the specimen out of the desired position.

If, however, the specimen must be studied under the high power objective $\left(\mathrm{X}_{440}\right)$ of a microscope, a cover slip is essential. For study under an oil immersion objective $\left(\mathrm{X}_{970}\right)$ the part of the integument is detached, flattened and covered.

If a cover slip is required on a thick mount, it may be added at any time after the surface of the balsam is hard. A drop of xylol is put on the surface and a large drop of thin balsam is put on a cover slip. The cover slip is inverted and one edge is rested on the balsam mount; the opposite edge is supported by a dissecting needle while it is slowly lowered into position. The cover slip is then adjusted by needles dipped in xylol.

If material is scarce - and especially if there is only a single larva - low-power studies are completed before the mount is covered. When material is abundant both covered and uncovered mounts are prepared.

\section{Reversibility}

It is reassuring to realize that, after the integument has been cleaned, every step is reversible. The same may be said of any series of steps and even of the entire technique from cleaned exoskeleton to covered slide mount. Only one precaution is necessary: hydration (i.e., from $95 \%$ alcohol to stain) must be gradual.

\section{Perfectionism}

The most important advice we can give to beginners is to avoid perfectionism in making mounts of ant larvae. Not only is perfectionism harmful to the technician (frustration, waste of time), but it can be very damaging to the material. Whenever the conscientious worker has an almost-good-enough preparation, there is always the temptation to try to adjust it a little bit more. Since the balsam must be a little thicker than usual to hold the specimen in the new position, the risk of damage is therefore proportionately increased and the damage may well be irreparable.

It is far better to stop short of perfection in mounting and resort to correction of the position of the slide. Before drawing we make 
any slight correction that may be necessary by propping up an edge or a corner or an end of the slide on a small lump of plasticine.

\section{Storage}

Prepared slides of ant larvae are always stored flat in the horizontal position. To store on edge is to risk displacement of the specimen, since the interior of a thick balsam mount requires years to harden. Thin mounts may, of course, be stored in any position as soon as the edges of the cover slip are sealed.

Preserved larvae may be stored indefinitely in $85 \%$ alcohol. The only precaution required is to prevent drying by evaporation through a faulty seal. (Dried larvae may be relaxed by methods given above.)

\section{Summary of Technique}

1. Puncture integument

2. $10 \% \mathrm{KOH}$ until clean

3. Distilled water - 15 minutes

4. Transfer to $1 \% \mathrm{HCL}$

5. Add 5 drops acid fuchsin; leave 12 hours

6. $95 \%$ alcohol for an hour

7. Add carbolxylol gradually
8. Carbolxylol 15-60 minutes

9. Xylol

10. Add thin balsam gradually

11. Transfer to balsam on slide

12. Arrange in desired position

13. Examine daily; rearrange as often as necessary until desired position has been maintained for 48 hours

\section{Descriptions}

We have generally followed this plan in writing our specific descriptions of ant larvae: body shape; leg vestiges ; spiracles; integument; body hairs; head shape; antennae; head hairs; labrum; mandibles; maxillae; maxillary palp; galea; labium; labial palp; opening of sericteries; hypopharynx. To facilitate taking notes we have prepared and used a mimeographed form with a blank space under each of the above headings.

When a larva is to be described the prepared slides are studied under a stereomicroscope for low magnifications and a microscope for high magnifications.

Measurements are made by comparison with the squares of an eyepiece reticule ( $=$ net micrometer) which has been calibrated with a stage micrometer.

\section{Drawings}

All our drawings of ant larvae are made with the aid of a microscope. A reticule (= net micrometer) is placed in the eyepiece. This superimposes a grid of squares on the image of the object. The drawing of the object is made on coordinate paper. The size of squares used is determined by the desired enlargement of the drawing. If 
coordinate paper is not available or if the squares are not of the desired size, it is not difficult to construct a coordinate grid on plain paper.

When the eyepiece grid is properly placed with reference to the image and the coordinate system is established on the paper, we are ready to start drawing.

The dimensions and proportions of the drawing are established by short marks across the lines of the coordinate system in the appropriate places. The main outline is constructed by joining these intersections with freehand lines. Structures inside or outside the main outline are treated in approximately the same way, but more freehand drawing is required because fewer squares are involved and smaller parts are likely to be wholly inside a single square. It is not necessary to complete all fine details at this stage.

The drawing is placed on a tracing table, a sheet of thin drawing paper (e.g., Ledger Linen) is laid on it; the drawing is copied onto the drawing paper in India ink with curve-pen (Keuffel and Esser). Finer details are drawn with a Hunt Mapping Pen No. IO4.

In the case of bilaterally symmetrical structures (e.g., head in anterior view) only one half is drawn, with a vertical matching line down the middle. After this half (but not the matching line) has been inked the pencil drawing is turned over on the tracing table, matched up to the first half and then inked.

Most of our drawings are orthographic projections of opaque objects (even though the integuments studied are actually transparent). Only structures on the near side of the plane of the outline are drawn. For example, in a side view of a larva the outline represents the imaginary middorsal and midventral lines; hairs and other parts attached beyond (= below, in the field of the microscope) the plane of these two lines are not shown. To our rule of assumed opacity a few exceptions are made, e.g. dark-colored structures overlaid by translucent tissues.

We have found $\mathrm{I} 20 \mathrm{~mm}$ to be a convenient length for a drawing of the entire larva. The plane of projection includes the middorsal line (imaginary), the midventral line (imaginary), the middle of the anus and the middle of the bottom of the labium. It is conventional to view the larva from the left. But if for any reason it has been drawn from the right, the original drawing (on co-ordinate paper) can be turned over on the tracing table before inking.

The head is drawn in full-face (i.e., anterior) view. The plane of projection passes through the uppermost line (imaginary) of the 
occipital border and the lowermost part of the ventral surface of the labium. A convenient width (at the widest place) is $75 \mathrm{~mm}$.

The mandible is also drawn in anterior view. The plane of projection passes through the apex, the inner ( $=$ medial) condyle and the outer (= lateral) condyle. If the mandible is drawn from a head mounted for full-face view, it is usually necessary to prop up an edge of the slide to bring these three points into the same horizontal plane. A convenient length for the mandible drawing is $65 \mathrm{~mm}$. 

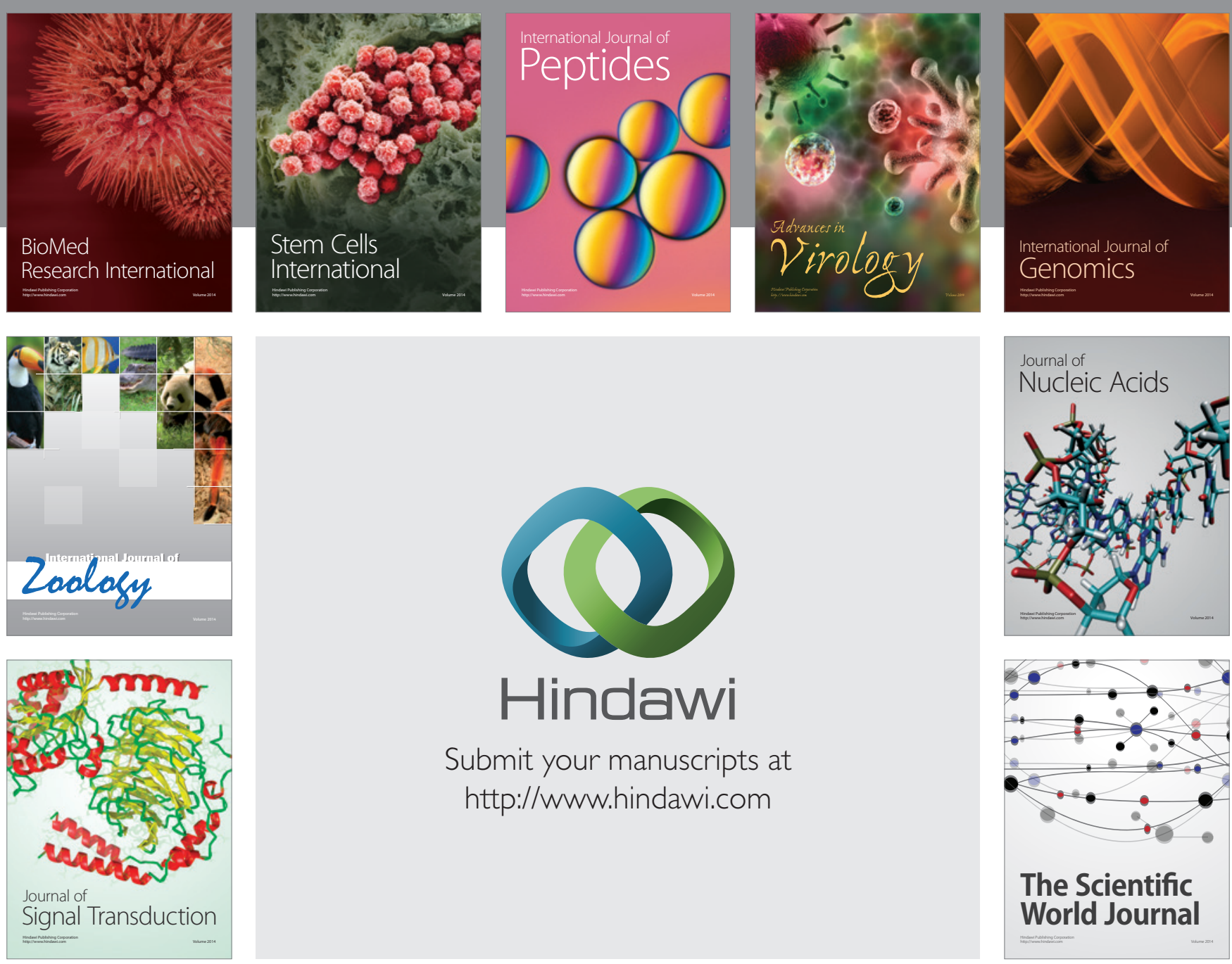

Submit your manuscripts at

http://www.hindawi.com
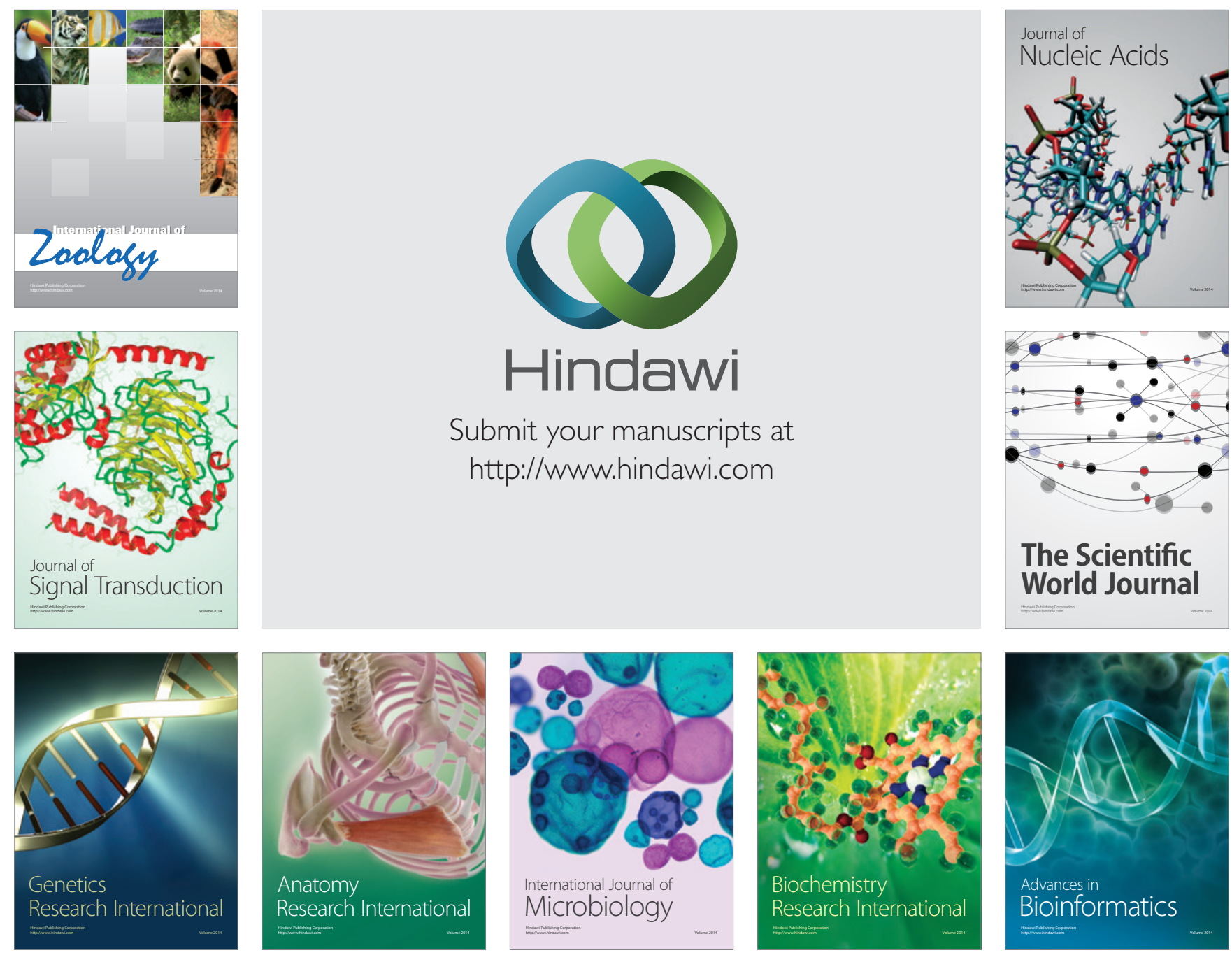

The Scientific World Journal
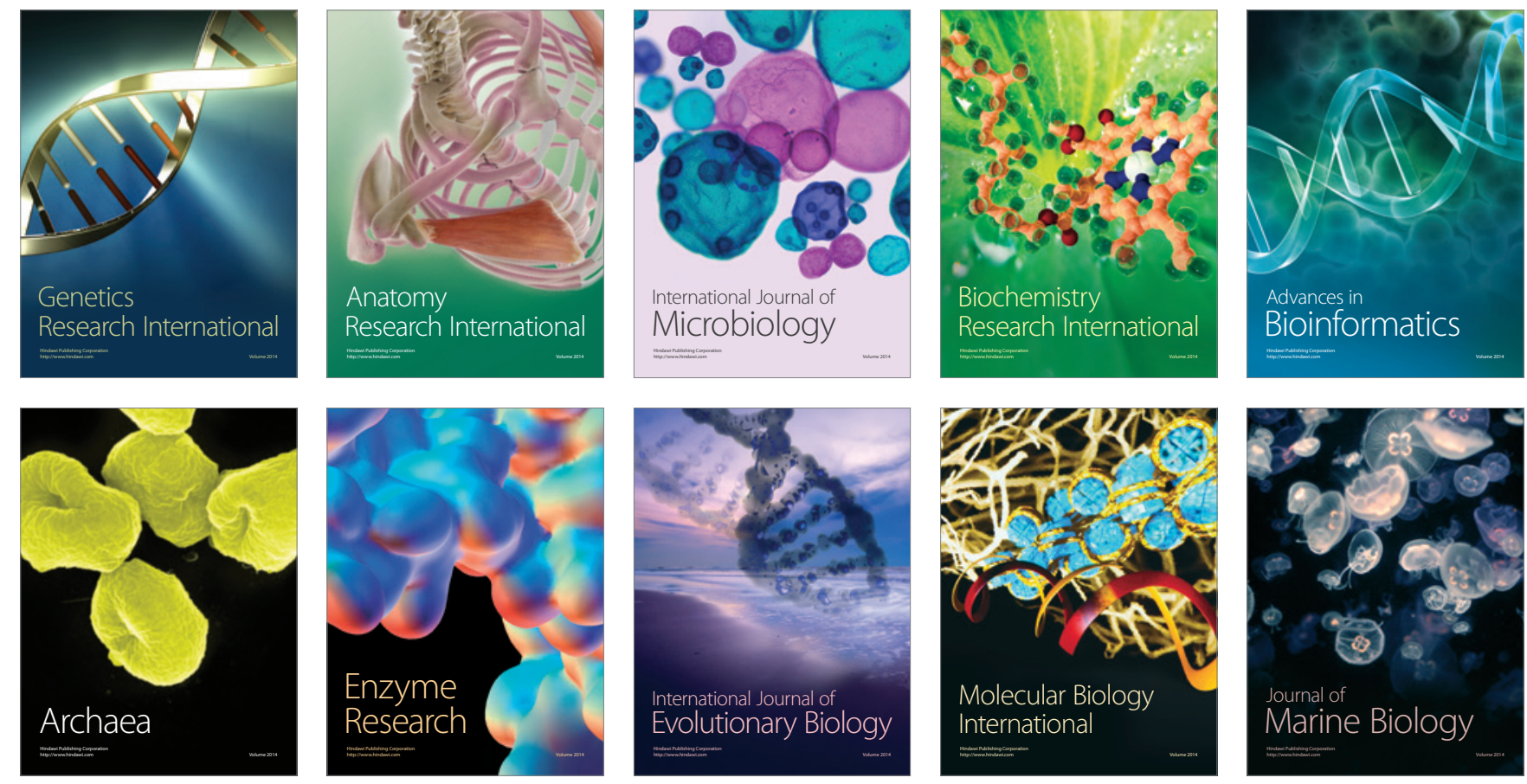